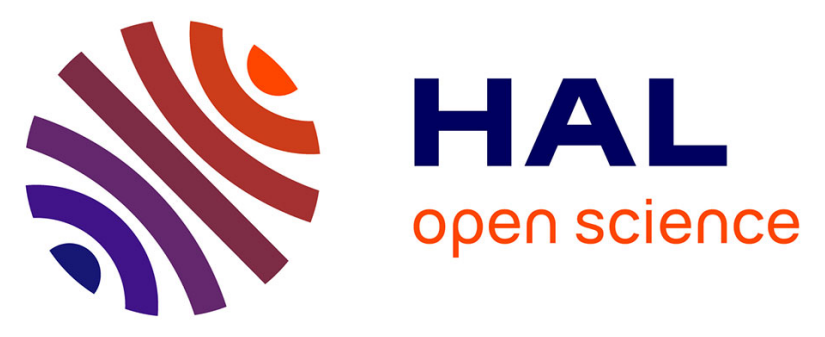

\title{
Simultaneous positron emission tomography and ultrafast ultrasound for hybrid molecular, anatomical and functional imaging
}

Jean Provost, Anikitos Garofalakis, Joevin Sourdon, Damien Bouda, Béatrice Berthon, Thomas Viel, Mailyn Perez-Liva, Charlotte Lussey-Lepoutre, Judith Favier, Mafalda Correia, et al.

\section{To cite this version:}

Jean Provost, Anikitos Garofalakis, Joevin Sourdon, Damien Bouda, Béatrice Berthon, et al.. Simultaneous positron emission tomography and ultrafast ultrasound for hybrid molecular, anatomical and functional imaging. Nature Biomedical Engineering, 2018, 2, pp.85-94. 10.1038/s41551-018-0188-z . hal-01864270

\section{HAL Id: hal-01864270 \\ https://hal-univ-paris.archives-ouvertes.fr/hal-01864270}

Submitted on 29 Aug 2018

HAL is a multi-disciplinary open access archive for the deposit and dissemination of scientific research documents, whether they are published or not. The documents may come from teaching and research institutions in France or abroad, or from public or private research centers.
L'archive ouverte pluridisciplinaire HAL, est destinée au dépôt et à la diffusion de documents scientifiques de niveau recherche, publiés ou non, émanant des établissements d'enseignement et de recherche français ou étrangers, des laboratoires publics ou privés.

\section{(1) (1) $\$$}

Distributed under a Creative Commons Attribution - NonCommercial - NoDerivatives 44.0 


\title{
Simultaneous positron emission tomography and ultrafast ultrasound for hybrid molecular, anatomical and functional imaging
}

\author{
Jean Provost $\oplus^{1}$, Anikitos Garofalakis ${ }^{2,3}$, Joevin Sourdon ${ }^{2,3}$, Damien Bouda ${ }^{2,3}$, Béatrice Berthon', \\ Thomas Viel ${ }^{2,3}$, Mailyn Perez-Liva ${ }^{2,3}$, Charlotte Lussey-Lepoutre ${ }^{2,4,5}$, Judith Favier ${ }^{2,3}$, \\ Mafalda Correia', Mathieu Pernot ${ }^{1}{ }^{1}$, Johanna Chiche ${ }^{6,7}$, Jacques Pouysségur ${ }^{8,9}$, Mickael Tanter $^{1 \star}$ and \\ Bertrand Tavitian ${ }^{2,3,10 *}$
}

Positron emission tomography-computed tomography (PET-CT) is the most sensitive molecular imaging modality, but it does not easily allow for rapid temporal acquisition. Ultrafast ultrasound imaging (UUI)-a recently introduced technology based on ultrasonic holography-leverages frame rates of up to several thousand images per second to quantitatively map, at high resolution, haemodynamic, biomechanical, electrophysiological and structural parameters. Here, we describe a pre-clinical scanner that registers PET-CT and UUI volumes acquired simultaneously and offers multiple combinations for imaging. We demonstrate that PET-CT-UUI allows for simultaneous images of the vasculature and metabolism during tumour growth in mice and rats, as well as for synchronized multi-modal cardiac cine-loops. Combined anatomical, functional and molecular imaging with PET-CT-UUI represents a high-performance and clinically translatable technology for biomedical research.

\begin{abstract}
$\mathrm{n}$ vivo imaging is a major driver of biomedical sciences, as well as an important pillar of evidence-based medicine for diagnosis, follow-up of disease evolution and assessment of treatment effi$\mathrm{cacy}^{1}$. In contrast with most bioanalytical techniques, in vivo imaging is non-invasive and non-destructive, and therefore repeatable. It can also be directly translated clinically, but comes with increased cost and complexity and typically yields just one or a few biological parameters at a time. This low parametric output is far from the multi-parametric data derived from omics technologies and greatly limits the capacity of in vivo imaging to decipher complex diseases with multiple hallmarks, such as cancer ${ }^{2}$. A preferred approach to overcoming this limitation is to combine different imaging techniques that superimpose co-registered information from the same subject. Accordingly, modern imaging calls increasingly on bimodal instruments, such as positron emission tomography (PET)-computed tomography $(\mathrm{CT})^{3}$, single-photon emission computed tomography-CT $(\mathrm{SPECT}-\mathrm{CT})^{4}$ or the more recent PET-magnetic resonance imaging $(\mathrm{MRI})^{5,6}$.

We propose a hybrid imaging instrument and approach based on PET-CT and ultrafast ultrasound imaging (UUI) ${ }^{7-10}$ to link metabolic activity with the physiological correlates described by haemodynamic, biomechanical, electrophysiological and quantitative structural parameters. PET is the gold standard for molecular imaging because it can detect picomolar concentrations of molecular probes with a resolution typically in the millimetre range. PET-CT
\end{abstract}

combines anatomical and molecular imaging modalities and is the reference clinical imaging technique for staging cancer extension. Other applications of PET include the diagnosis and follow-up of Alzheimer's disease ${ }^{11}$, differential diagnosis of neurological disorders $^{12}$ and identification of vulnerable atheroma plaques ${ }^{13}$. The exquisite capacity of PET to non-invasively depict local biochemistry has triggered the invention of myriad PET tracers (referenced in the Molecular Imaging and Contrast Agent Database ${ }^{14}$ ), of which an increasing number reach clinical approval for diagnostics.

UUI has the unique capability of acquiring images in a large field of view within a fraction of a millisecond, which enables the study of rapid phenomena and the accumulation of large amounts of data to achieve high sensitivity. UUI benefits from a very active field of research from which imaging modes emerge on a regular basis $^{15}$. Applications include the imaging of brain activation ${ }^{7,9,10}$, quantification of tissue stiffness for cancer or fibrosis staging ${ }^{16}$, cardiac activation ${ }^{17}$, fat content ${ }^{18}$, mechanical stress ${ }^{19}$ and non-invasive angiography of small vessels ${ }^{20}$ in vivo. Most recently, UUI has been extended to three dimensions, allowing for the acquisition of thousands of volumes per second ${ }^{21}$, and the introduction of a super-resolution UUI mode non-invasively revealed the anatomy and the fully quantified function of resolved capillaries of the entire rat brain in vivo and on the microscopic scale ${ }^{8}$. UUI is also highly translational since imaging modes developed for small animals can be readily applied clinically using adapted ultrasound probes.

\footnotetext{
'Institut Langevin, Ecole Supérieure de Physique et de Chimie Industrielles, Paris Sciences and Letters Research University CNRS UMR 7587 Inserm U979, Inserm Technology Research Accelerator in Biomedical Ultrasound, Paris, France. ${ }^{2}$ Inserm, UMR970, Paris Cardiovascular Research Center, Paris, France. ${ }^{3}$ Faculté de Médecine, Université Paris Descartes, Sorbonne Paris Cité, Paris, France. ${ }^{4}$ Faculté de Médecine, Université Pierre et Marie Curie, Paris, France. ${ }^{5}$ Nuclear Medicine Department, Pitié-Salpêtrière Hospital, Paris, France. ${ }^{6}$ Faculté de Médecine, Université de Nice Sophia Antipolis, Nice, France. ${ }^{7}$ Équipe Contrôle Métabolique des Morts Cellulaires, Inserm, U1065, Centre Méditerranéen de Médecine Moléculaire, Nice, France. ${ }^{8}$ Institute for Research on Cancer and Aging, Université de Nice Sophia Antipolis, Centre Antoine Lacassagne, Nice, France. ${ }^{9}$ Department of Medical Biology, Centre Scientifique de Monaco, Monaco, Monaco. ${ }^{10}$ Department of Radiology, Georges Pompidou European Hospital, Paris, France. Jean Provost and Anikitos Garofalakis contributed equally to this work. Mickael Tanter and Bertrand Tavitian jointly supervised this work. *e-mail: mickael.tanter@espci.fr; bertrand.tavitian@inserm.fr
} 
We present a PET-CT-UUI triple-imaging modality that integrates the three imaging modalities in one device for simultaneous, fully co-registered imaging, enabling image fusion independent of motion and without increasing acquisition times. The PET-CTUUI instrument was assembled from existing, commercially available devices using lightweight and portable UUI instrumentation for which dedicated, customized sequences were developed. We show that full, markerless, rigid-body three-dimensional (3D) registration was accurately achieved by controlling the ultrasound probe with a six-degrees-of-freedom motorized micropositioner and that the presence of the ultrasound probe had a negligible impact on the quality of the PET volumes. Remarkably, PET-CT-UUI can produce multi-parametric information that is currently unobtainable with any other non-invasive imaging method. Here, we demonstrate in pre-clinical oncology and cardiology applications the capability of this imaging modality to yield unprecedented information from fused, simultaneously acquired volumes of PET-CT-UUI images.

\section{Results}

Figure 1 shows the processing steps and experimental set-up developed for PET-CT-UUI. First, the UUI transducer was positioned using a motorized micropositioner (Fig. 1a) over the organ of the mouse or rat to be imaged using real-time B-mode imaging. Then, the animal bed was moved, together with the UUI transducer, inside the PET-CT gantry and an initial CT scan was acquired. Acquisition of a UUI volume was achieved by displacement of the ultrasound probe using a six-degrees-of-freedom motorized micropositioner remotely controlled in programmed steps, for which the coordinates were expressed as a function of the PET-CT system coordinates. This set-up enabled fiducial-marker-free affine coregistration with an estimated accuracy equal to $0.095 \pm 0.028 \mathrm{~mm}$ and based on a single calibration procedure that can be re-used for any number of experiments. Full-body, four-dimensional, 60-minlong dynamic list-mode PET scans (Fig. 1b) were acquired after intravenous administration of $2^{\prime}$-deoxy- $2^{\prime}-\left({ }^{18} \mathrm{~F}\right)$ fluoro-D-glucose (FDG). The effect of the presence of the ultrasound probe in the gantry during PET acquisitions was quantified and deemed negligible, which enabled the simultaneous scanning of organs of interest in one or several UUI modes: B-Mode, Doppler (Fig. 1b) or shear-wave elastography. Experiments were conducted on tumours in mice and on the hearts of rats, and specific methodologies were developed for each case. Following 3D image reconstructions, the CT, PET and UUI volumes were re-sampled to the same voxel size and registered to yield a fused PET-UUI volume of the organ of interest (Fig. 1c and Supplementary Video 1). Mapped parameters derived from the image volumes; for example, peak, mean or maximal standard uptake values (SUVs) for FDG PET scans, and tissue density maps, perfused vessel density and tissue elasticity from UUI, were then analysed by local correlation analysis using bivariate histograms.

Here, we present selected examples that illustrate the unique sets of parameters whose topography can be derived from PET-CTUUI: (1) bimodal maps of energy metabolism and microcirculation during tumour development, (2) tumour vascularization patterns relative to the metabolic pathway followed by cancer cells (aerobic, anaerobic or mixed) and (3) the superimposition of cine-loops of glucose metabolism, intramyocardial vessels, wall motion and elasticity in the myocardium.

Topographical relationship between glucose metabolism and vascularization. Simultaneous fused PET-CT-UUI volumes of tumour metabolism and vascularization were obtained in mice $(n=8)$ implanted with tumoural cell lines and tumour allografts (Fig. 1c). Both the vasculature of the tumour and the FDG uptake were fully co-registered, yielding observations of the topographic relationship between vascularization and metabolism (Fig. 1c).

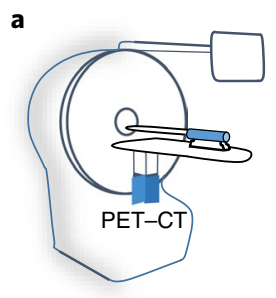

b
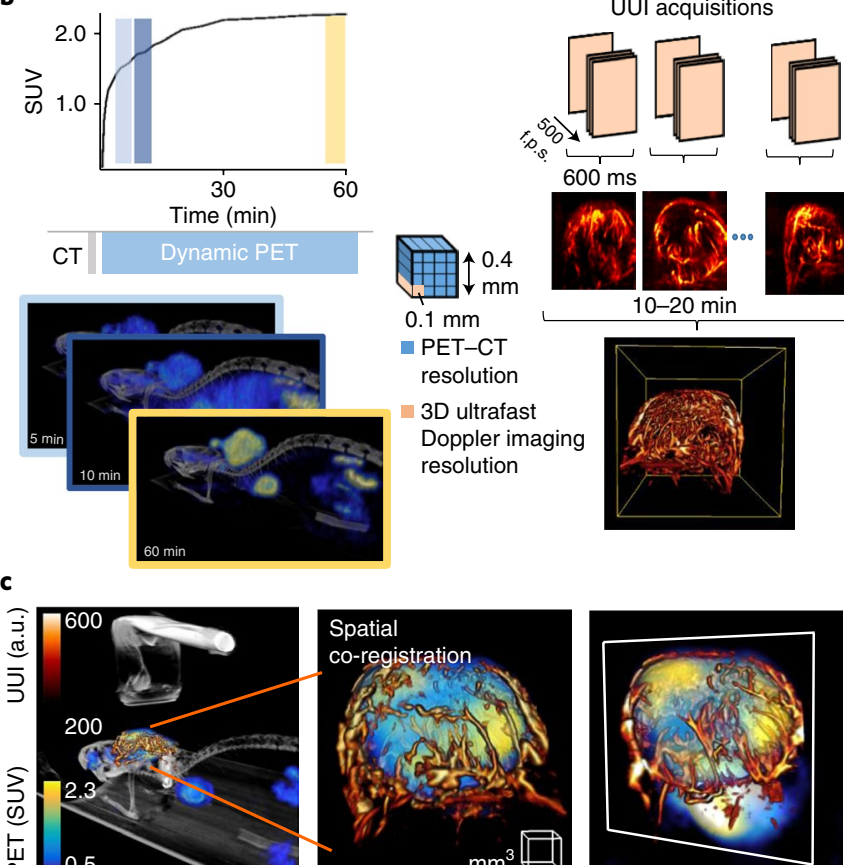

Fig. 1 | PET-CT-UUI set-up and acquisitions. a, The PET-CT-UUI system is a combination of an ultrafast ultrasound scanner and a PET-CT device. The coordinates of the ultrasound volume are determined by a motorized micropositioner, whereas the coordinates of the PET-CT volume are defined by the position of the animal bed. $\mathbf{b}$, Left: SUV for FDG PET scans (top, black line). After an initial CT scan, subsequent PET-CT acquisitions were performed in list mode, which allows for dynamic PET-CT 3D reconstructions of a mouse (bottom). The coloured bars (top) indicate the time points at which the images (bottom) were acquired. The PET-CT voxel size was $400 \mu \mathrm{m} \times 400 \mu \mathrm{m} \times 400 \mu \mathrm{m}$. Right: UUI in ultrafast Doppler imaging mode in a mouse tumour, which involves sequentially performing and processing parallel 600-ms-long UUI acquisitions at 500 frames per second (f.p.s.) to form a 3D volume of the tumour vasculature. The size of the volume depends on the probe and sequence used and the travel distance of the micropositioner, and was typically $20 \mathrm{~mm} \times 12.9 \mathrm{~mm} \times 20 \mathrm{~mm}$ for up to 200 positions. The reconstructed UUI voxel size was $100 \mu \mathrm{m} \times 100 \mu \mathrm{m} \times 100 \mu \mathrm{m}$. c, PET-CT-UUI images of a mouse tumour xenograft. After resampling both volumes to the UUI voxel size using spline interpolation, rendering of the fused data was performed using dedicated software, with three different colour scales: greyscale for CT, heat scale for UUI (Doppler arbitrary units, a.u.), and yellow-blue scale for PET (SUV). Left: the ultrasound probe located above the targeted tumour in the CT volume and the fused PET-UUI volume depicting the vasculature and FDG uptake. Middle: maximal intensity projection view of the spatially co-registered tumour. Right: view of image plane inside the volume.

Note, for instance, the large vessels entering the tumour area and their ramification in smaller vessel branches penetrating deeply inside the tumour regions with high FDG uptake. With the present setting, the diameter of the smallest vessels resolved using ultrafast Doppler analysis was approximately $100 \mu \mathrm{m}$. 
The topographic relationship between tumour vascularization and metabolism was explored longitudinally by repeated PET-CTUUI examinations of the same animals. A series of PET-CT-UUI volumes obtained during the growth of a tumour allograft transplanted from immortalized mouse chromaffin cells is shown in Fig. 2 and Supplementary Video 2. This model carries a knockout of the Sdhb gene, encoding the catalytic subunit of the mitochondrial enzyme succinate dehydrogenase. In humans, $S D H B$-deficient tumours are characterized by a strong avidity for glucose $\mathrm{e}^{22}$ and enhanced angiogenesis $^{23,24}$. While the growth of the tumour implanted in the dorsal fat pad of nude mice ${ }^{25}$ was accompanied by the development of tumour vessels and increased FDG uptake, fusion of PET with UUI (Fig. 2a) demonstrated that metabolism and vascularization did not follow parallel evolutions. On the contrary, quantitative parameters derived from the PET-UUI image volumes indicated that, during tumour growth, the ratio of perfused vessel volume over tumour volume decreased continuously from $51 \%(n=4)$ on day 14 to $28 \%$ $(n=7)$ on day 32 after implantation. Relative to the metabolic volume (that is, the area in which the SUV exceeds $30 \%$ of the peak value), the vascular volume underwent a threefold decrease (Fig. 2b) between 14 and 32 days after implantation $(P=0.02$, Wilcoxon
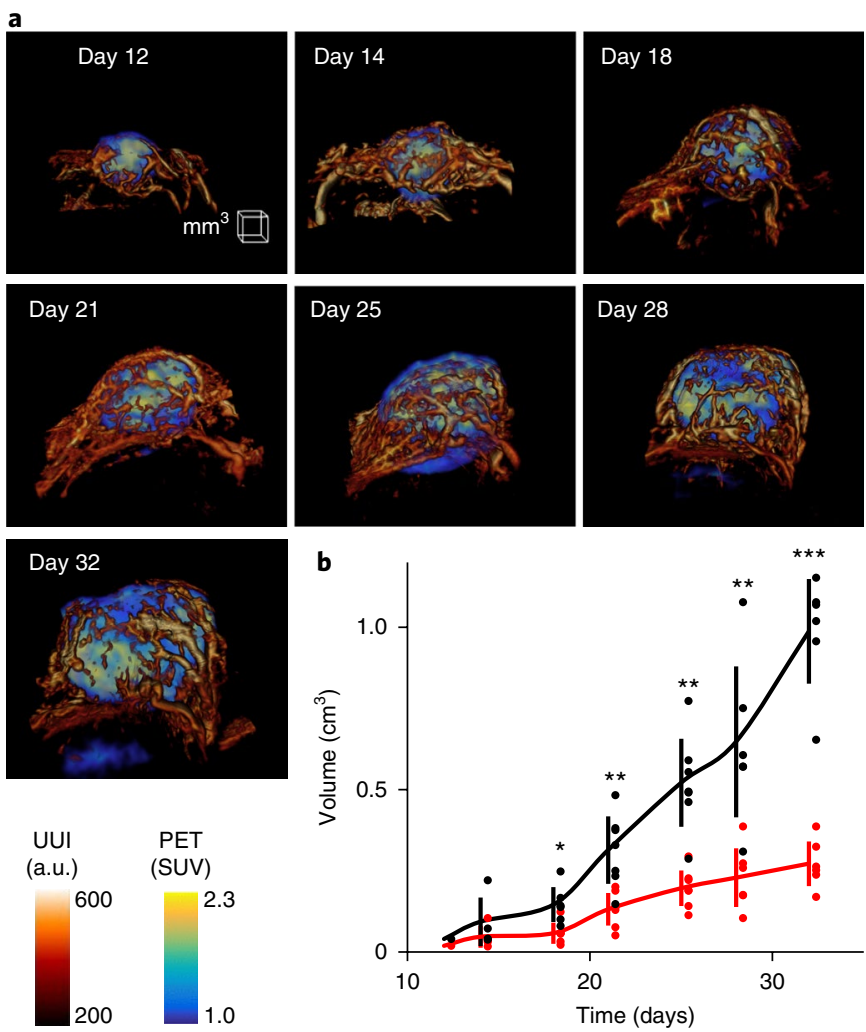

Fig. 2 | Longitudinal PET-UUl imaging monitors the evolution of metabolism and vascularization during tumour growth in a mouse.

a, Fused volumes of FDG uptake and perfused vessels of an Sdhb-deficient allografted tumour at indicated times post-implantation in the fat pad of a nude mouse. The images of perfused vessels were obtained using the ultrafast Doppler technique without the use of contrast agents and are depicted using the respective colour scales shown for PET (SUV) and UUI (Doppler arbitrary units, a.u.). b, Volumes of the segmented PETavid region corresponding to the total tumour volume (black line) and segmented perfused vessel region (red line) plotted against time after tumour implantation. The graph shows individual, mean and s.d. values of independent measures in 4 to 7 animals, except on day 12 , for which there was only one animal. Significant differences between the vascular and tumour volumes are indicated: ${ }^{\star} P<0.05 ;{ }^{\star \star} P<0.01 ;{ }^{\star \star \star} P<0.001$. signed rank test). Other parameters, such as the vessel length, tortuosity and number of nodes, may also be measured on the images and further correlated with tumour metabolism.

Bivariate analysis for tumour phenotyping. PET-CT-UUI was used for simultaneous non-invasive measurements of distinct tumour hallmarks ${ }^{2}$ in the same spatial coordinate system. Each individual voxel of the combined PET-CT-UUI dataset is defined by its spatial coordinates and by two quantitative parameters; for instance, (1) SUV of FDG, representing the deregulation of energy metabolism and (2) Doppler intensity, corresponding to functional, perfused blood vessels. In a reverse-genetic approach, mutants of the CCL39 tumour cell line (denoted wild type (wt)) were genetically engineered to abolish either glycolysis (gly- cells) or mitochondrial respiration (res ${ }^{-}$cells $)^{26-28}$ and allowed to grow subcutaneously in mice to a tumour volume of $400 \mathrm{~mm}^{3}$. Mitochondrial respiration describes metabolic processes occurring in the mitochondria and requiring oxygen to convert the energy stored in nutrients to adenosine triphosphate. Thus, the three tumours represented three different energetic metabolic statuses: an exclusively anaerobic metabolism ( res $^{-}$), an exclusively aerobic metabolism (gly ${ }^{-}$) and both aerobic and anaerobic metabolism (WT), all else being equal in their genetic background ${ }^{26-28}$. Figure 3 and Supplementary Video 3 show fused PET-UUI volumes (Fig. $3 \mathrm{a}-\mathrm{c}$ ) and their associated bivariate histograms (Fig. $3 \mathrm{~d}-\mathrm{f}$ ) of the voxel value distributions along a metabolic axis (FDG: SUVs) and a vascular axis (power Doppler signal intensity) of $w t(n=5)$, gly $^{-}(n=3)$ and res $^{-}(n=4)$ tumours. PET-CT-UUI unambiguously separated tumour types according to their metabolic and vascular profiles: compared with the $w t$ profile, purely glycolytic res ${ }^{-}$tumours exhibited a high FDG uptake with a low vessel density, while, in contrast, gly tumours exhibited a low FDG uptake and a high vessel density.

Time-varying PET-UUI for cardiac applications. The capacity of UUI to acquire images at high rates is particularly useful for imaging organs in rapid motion, such as the rat heart, which typically beats 300-400 times per minute. Figure 4 and the corresponding Supplementary Video 4 show the PET-UUI acquisition of a beating rat heart using a commercially available $12 \mathrm{MHz}$ transducer probe, used clinically for musculo-skeletal applications, connected to the PET-CT-UUI motorized arm by way of a custom 3D-printed adaptor. The UUI B-mode acquisitions provided a cine-loop of the position of the beating heart on which the PET maps of the FDG uptake in the myocardium were precisely superimposed (Fig. 4a). This hybrid image allowed for the accurate delineation of the cardiac anatomy using UUI. Additionally, the UUI Doppler mode was used to map large intramyocardial vessels (Fig. 4b), which is an indirect marker of perfusion ${ }^{29}$, and the shear-wave elastography mode was used to quantify the myocardial stiffness during the entire cardiac cycle (Fig. 4c), of which the diastolic value could become a potential quantitative marker for viability ${ }^{30,31}$.

\section{Discussion}

Ever since the end of the seventeenth century, when Sir Robert Hooke and Antoni van Leeuwenhoek observed living cells under a microscope, progress in imaging instrumentation has fostered our understanding of the living world and escorted-and sometimes driven-medical diagnosis and treatment follow-up. In contrast, there is a strong trend in today's biological research and medicine to address the complexity and multidimensionality of living systems using high-throughput methods such as the omics technologies or multispectral fluorescence, while in vivo imaging struggles to produce meaningful multi-parametric information. The complexity and cost of non-invasive biomedical imaging instruments and the limited number of quantitative imaging biomarkers that can be derived from any one technique greatly limit the capacity of 


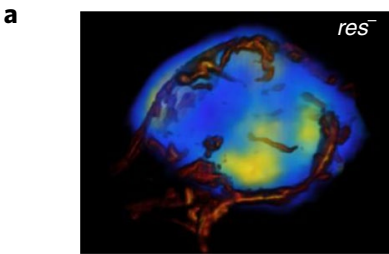

b

d

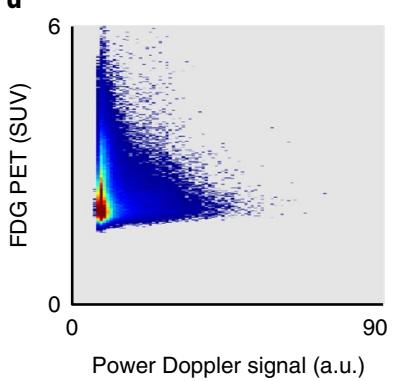

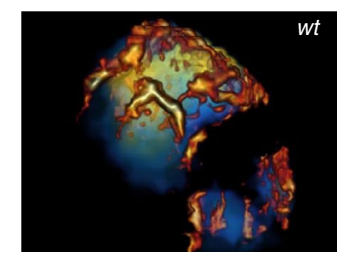

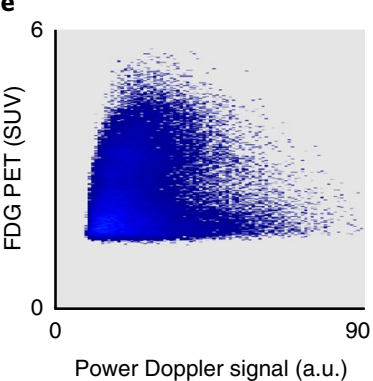

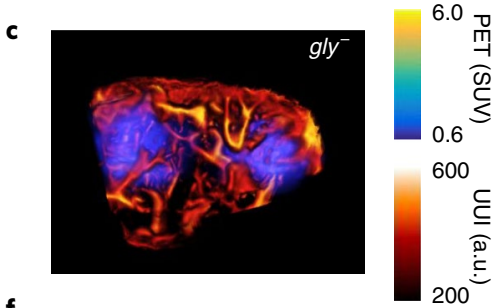

f

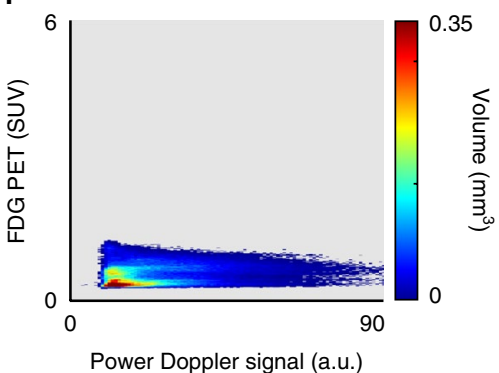

Fig. 3 | Phenotyping tumours in mice on the basis of bivariate, FDG-ultrafast-power-Doppler histograms. a-c, Fused images of FDG uptake and perfused vessels in tumours grown subcutaneously in nude mice with three variants of the Ras-transformed CCL39 cell line. Left to right: a respiration-deficient mutant (res; a), the WT CCL39 (wt; b) and a glycolysis-deficient mutant ( $g l y ; \mathbf{c})$. d-f, Bivariate histograms corresponding to panels a-c depict the relationship between the pixel-wise SUV and power Doppler signal proportional to glucose metabolism and blood volume, respectively. The heat map indicates the tumour volume characterized by each of the PET-UUI value pairs. Figure shows typical cases from triplicates for each mutant.

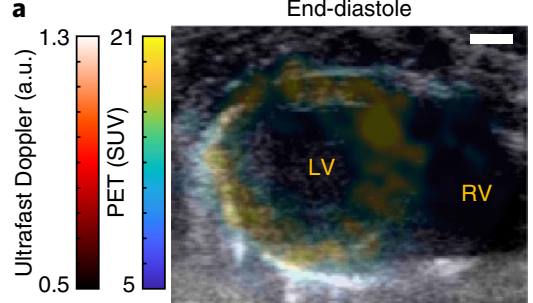

b

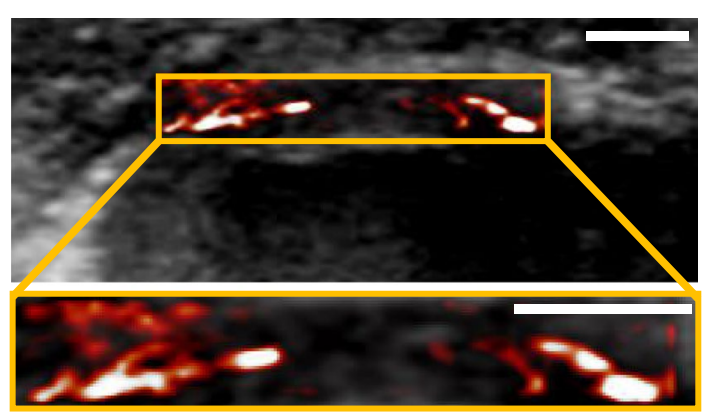

End-systole
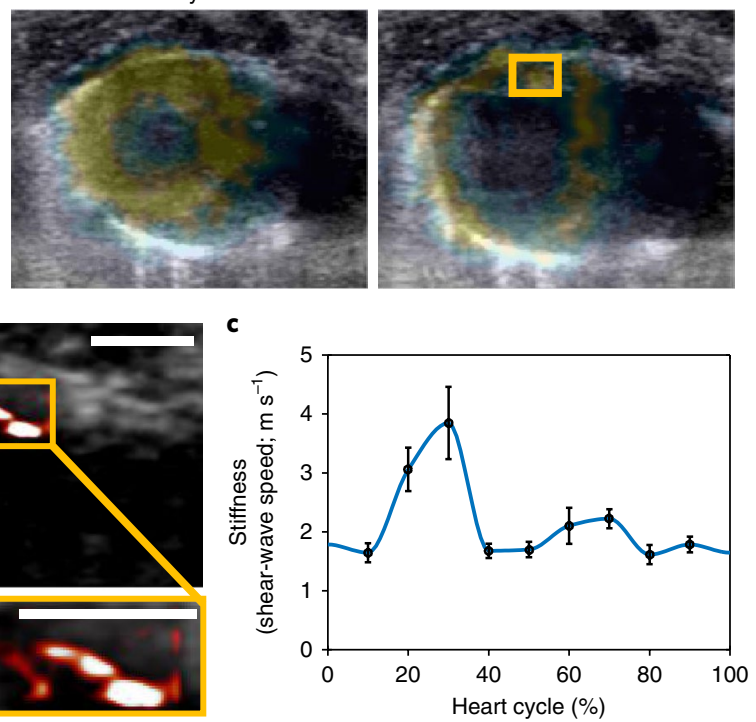

Fig. 4 | PET-CT-UUI of the beating rat heart in a short-axis view. a, Selected time windows of a beating rat heart showing an overlay of the heart wall and the metabolic activity of the myocardium in a short-axis view at end-diastole (left), end-systole (middle) and mid-diastole (right). Note the good agreement between the position of the metabolic signal in FDG PET and the myocardial wall depicted by the UUI B-mode images. Each image corresponds to the mean signal during one-eighth of a heart cycle. Shown are typical images of one animal from ten replicates. $\mathbf{b}$, Ultrafast Doppler images of perfused intramyocardial vessels - a potentially useful parameter in assessing myocardial perfusion. c, Myocardial shear-wave velocity-a quantitative biomarker for myocardial stiffness-measured using shear-wave imaging in the region of interest indicated by the yellow rectangle in a as a function of time. The increase in shear-wave velocity during systole is correlated with the increase in the stiffness of the myocardium. Data in $\mathbf{b}$ and $\mathbf{c}$ were obtained in one rat in the region of interest shown in $\mathbf{a}$. In c, the error bars correspond to the s.d. within the region of interest indicated by the yellow box in $\mathbf{a}$. LV, left ventricle; $\mathrm{RV}$, right ventricle. Scale bars, $2 \mathrm{~mm}$.

imaging to produce multiplexed simultaneous analyses of living individuals. A favoured approach is the development of hybrid imaging instruments, such as PET-CT or PET-MRI, which in turn come with increased complexity and cost. Therefore, it is tempting to call on the relatively inexpensive and portable ultrasound technology to bring more information to other imaging modalities. Moreover, similar to PET-MRI, but not to PET-CT, image acquisition with
PET-UUI is simultaneous, allowing the near-perfect co-registration that is necessary to establish a topographical relationship between multiple imaging parameters, and the rate of acquisition of UUI is fully compatible with the imaging of moving organs; for example, the beating hearts of small rodents. From a medical perspective, the main disadvantage of ultrasonography is the lack of full-body imaging, but, in our view, this is largely compensated for by the exclusive 
images obtained in the UUI modes that offer spatial and temporal resolutions unmatched by any other in vivo imaging technique in equivalent fields of view. Moreover, recent ultrasound research has tremendously enriched the capability of this modality to provide multiple independent physical parameters, such as blood flow in small vessels ${ }^{7,8}$, blood perfusion ${ }^{32}$, mechanical properties such as shear modulus and shear viscosity ${ }^{16}$, muscle contractility ${ }^{31}$, fibre tractography ${ }^{33}$ and neurovascular brain activity ${ }^{7}$ UUI is a genuine multi-parametric technology ideally complemented by PET molecular imaging. The limitations of UUI include limited tissue contrast in anatomical imaging (B-mode), user-dependence and a lack of four-dimensional imaging. The latter two limitations were partially lifted in this study by the use of a motorized micropositioner and are expected to be further addressed in the future by improvements in matrix-array technology ${ }^{21}$

Here, we demonstrate the feasibility of co-registered and simultaneous PET-CT-UUI imaging in vivo. This hybrid imaging was implemented without major hardware modifications of commercial imaging instruments. PET and UUI images were automatically fused without human input and with a precision in co-registration much better than the spatial resolution of any PET system. The presence of the ultrasound probe inside the PET gantry had a modest impact on gamma ray attenuation with little visible effect on the PET images. High-resolution UUI images were obtained in vivo in rodents with a full range of UUI modes: B-mode for tissue anatomy, ultrafast Doppler mode for microcirculation and shear-wave imaging for tissue elasticity. All UUI images were fully co-registered with images of FDG uptake in tissue. A simultaneous PET-CT-UUI design has many practical and fundamental advantages, in contrast with other hybrid imaging technologies for which the sequential design is often easier to implement than the simultaneous one. For example, in the cases of PET-CT and PET-MRI, both modalities are non-contact and performed inside a gantry, which enables the use of the same animal bed that can be inserted into one or the other device. Moreover, in the case of PET-CT, the added modality is often used to provide anatomical information, which is essentially insensitive to the physiological state of the animal. In PET-CTUUI, neither of these advantages is present in a sequential system. A UUI scan requires contact between the animal and a coupling device, which leads to tissue non-rigid deformation that is difficult to account for using sequential co-registration. Moreover, many UUI modes are physiological rather than anatomical; for example, ultrafast Doppler imaging maps the blood volume circulating in perfused vessels, which is sensitive to the anaesthesia level and body temperature. In a simultaneous design, this sensitivity is a benefit, as it reflects the vasculature that transported the PET contrast agent at a given time, while in a sequential design it would degrade coregistration, leading to unacceptable uncertainty, for example, in the bivariate histograms of Fig. 3. Similar arguments can also be made regarding cardiac physiology, in which multiple parameters can change between two sequential acquisitions, such as ejection fraction, wall perfusion or heart rate. A simultaneous design is hence, in many ways, more straightforward (simple affine co-registration can be used), more accurate (physiology is consistent in both scans) and saves experimental time (acquisitions are performed concurrently). A remarkable application of simultaneous PET-UUI would be the measurement of transient or aperiodic signals that occur outside a regular time frame. Examples of such applications include mapping brain activity with both modalities, as has been done using PET-MRI ${ }^{5}$, or the co-measurement of time-dependent cardiac perfusion using UUI and dynamic FDG uptake using PET. As an example, the whole-brain spatiotemporal dynamics of epileptic seizures can be uniquely assessed using the UUI modality due to its very high sensitivity ${ }^{7}$. Other examples include transient ischaemicreperfusion events in the intramyocardial vasculature, which is accessible with $\mathrm{UUI}^{34}$.
Here, the potential interest of combining exquisitely sensitive molecular information with high-resolution anatomical and physiological information was demonstrated in proof-of-concept studies in oncology and cardiology. We show simultaneous 3D images of a tumour's metabolism together with its vascularization, as well as the evolution of the relationship between metabolism and vasculature during tumour growth across longitudinal PET-CT-UUI acquisitions in the same animals. We also demonstrate that co-registered measurements of both vasculature and metabolism stratify different types of tumours on the basis of these two major hallmarks of cancer. Cancer cells can adapt their metabolism to the concentrations of oxygen and glucose inside the tumour and can also direct the formation of new blood vessels by triggering angiogenesis, which in turn makes oxygen and glucose available ${ }^{35,36}$. This implies the existence of tight links between the deregulation of energy metabolism and the abnormal vasculature typical of tumour tissues, and creates local heterogeneity inside tumours ${ }^{35}$. In addition, a major mechanism of evasion from anti-angiogenic treatment is the switch from aerobic to anaerobic metabolism ${ }^{37}$. It is therefore of great theoretical and practical interest to determine-in vivo and non-invasivelythe metabolic and vascular profiles of tumours ${ }^{1,2}$. It can be envisioned that topographical analysis of these two hallmarks will yield a combination of tumour biomarkers with potential impact for in vivo cancer phenotyping, and help in the optimization of selective co-targeting of mechanism-guided therapeutic combinations for more effective and durable cancer therapies ${ }^{2}$.

Co-registered images of the anatomy and myocardial stiffness combined with images of local FDG uptake in the beating rodent heart are a proof-of-concept of the potential of our PET-CT-UUI system for cardiac imaging. The capability to follow the motion of the heart in real time allows for a true delineation of the myocardial FDG uptake based on the dynamic cardiac anatomy. Shear-wave elastography and ultrafast Doppler imaging provide an approach to explore myocardial viability in terms of myocardial stiffness ${ }^{30,31}$ and perfused intramyocardial vessels. In the future, other UUI modes for cardiac applications will be implemented to map perfusion ${ }^{32}$, the activation sequence of the heart ${ }^{17}$, local deformations ${ }^{38}$ and even cardiac muscle contractility ${ }^{30,31}$. In conjunction with FDG uptake, these parameters could become powerful biomarkers to assess myocardial viability.

Whenever a new hybrid imaging instrument is introduced, it is tempting to speculate on its innovating potential and its capacity for breakthroughs. In its actual state, the pre-clinical PET-CT-UUI prototype presented here can ideally explore the physiology and pathophysiology of organs in rodents with high temporal and spatial resolution. Imaging of the heart and tumours was shown here as an example, but it is also possible to image other organs, such as the liver, kidneys and brain, as we ${ }^{39}$ and others ${ }^{35}$ have shown in previous reports using separate PET and UUI instruments. Regarding tumour imaging, one of the applications of bivariate mapping of circulation and metabolism is to explore metabolic symbiosis ${ }^{37}$, which was recently found to be responsible for evasion from anti-angiogenic therapy ${ }^{36}$, and a longitudinal exploration of the effect of sunitinib on tumour microcirculation and metabolism is currently under way in our laboratory. PET-CT-UUI is ideal to test the concerted action on metabolism and vascularization of chemotherapeutic drugs and drug candidates, or of their combinations, as well as the outcome of radiotherapy or radiotherapy/chemotherapy combinations in various animal models of cancer. Another straightforward application would be to assist the development and validation of PET radiotracers and radiopharmaceuticals by monitoring the role of tumour vascularization on their tissue uptake, with useful applications for the compartmental modelling of dynamic PET data. From a fundamental perspective, the acquisition modes that we recently developed $^{8}$ to resolve vessels down to a diameter of approximately $10 \mu \mathrm{m}$ open perspectives for research in the exchanges between capillaries 
and tissue ${ }^{40}$. The near-perfect superimposition of image volumes offers the attractive option to follow rapidly moving organs such as the heart, and longitudinal studies of the metabolic, functional and mechanical (that is, tissue elasticity) consequences of myocardial ischaemia are under way.

In the same way as simultaneous PET-MRI, the first implementation of simultaneous PET-CT-UUI was developed in a pre-clinical setup, but it can be adapted to yield a clinical instrument. We show here that the integration of an ultrasound scanner in an existing PET-CT was performed at relatively low cost and without any major changes to the instrument's hardware. The introduction of UUI probes in the larger bores of clinical PET gantries is likely to be easier than in the narrow pre-clinical ones. The interactions between the $511 \mathrm{keV}$ gamma rays of the annihilation photons and the ultrasound probe are limited, as was confirmed by the results shown herein and by other studies ${ }^{41}$. The wide availability of adapted probes can be leveraged so that the same electronics can be used for both pre-clinical and clinical studies. UUI modes are rapidly emerging and allowing for exquisite images of the vasculature and unique measurements, such as rapidly varying myocardial stiffness, which is appealing for cardiac studies. In oncology, it is reasonable to envisage using PET full-body images to define the image volume targets for UUI, but the limited field of view of UUI is an important limitation, which would be problematic during exploratory scans and for four-dimensional imaging of moving organs. Nevertheless, solutions involving motorized arms and matrix arrays can be envisioned with reasonable additional time and effort. The benefit of such co-registration would be important as, at present, imaging volumes are acquired during different exams, slowing the patient workflow, and in unmatched positions of the patient's organs, which, in many cases, such as in breast imaging, condemns co-registration. As UUI is a modality for which clinical applications are still in early phases of development, the specific clinical applications for PET-CT-UUI remain naturally speculative at this stage. However, they involve a large array of organs as UUI can be applied to any organs accessible with conventional ultrasound imaging. Typically, the deeper the organ is situated the lower the ultrasound frequency used, and the lower the frequency the worse the spatial resolution. In the worst case, the upper limit of resolution is typically on the order of $500 \mu \mathrm{m}$ (corresponding to a $3 \mathrm{MHz}$ centre frequency), which is better than the inherent resolution of PET and comparable to CT and MRI spatial resolutions. Reasonable scenarios for tumour characterization will be to apply the biomarkers offered by UUI, such as tissue stiffness and vascularization, for improved personalized monitoring and treatment. Importantly, UUI-based motion correction is likely to improve PET image resolution in organs with limited motion, and to reduce acquisition times in the heart by limiting the need for electrocardiogram (ECG)-gatingbased motion correction in standard cardiac PET scans.

Ultrasound technologies are also particularly well-suited for the development of theranostic approaches, since ultrasound waves can be used for both imaging and therapy in the form of histotripsy, lithotripsy, high-intensity focused ultrasound and drug delivery, to name a few. The synergy between PET and ultrasound could thus extend towards the therapeutic field. Finally, it has not escaped our attention that the synergy of the spatiotemporal co-registration could also be used to overcome some of the current limitations of PET technology (for example, ultrasound-based motion correction $^{41}$ or correction of the biases in measuring arterial input functions) and for the integration of UUI maps in dynamic PET compartmental models. A potential benefit of simultaneous acquisition is ultrasound-based motion correction to enhance the PET reconstruction. Ultrasound is particularly apt for this task as it can measure motion of a few micrometres, and high frame rates enable better accuracy and precision ${ }^{42,43}$. Motion correction of PET imaging using conventional ultrasound has been shown before ${ }^{41}$ and UUI is expected to provide equal or better performances due to its higher frame rates, as shown for other applications such as localization microscopy ${ }^{44}$, heart wall tracking and cardiac strains $s^{45,46}$, as well as ultrasound image formation ${ }^{47}$.

In summary, the PET-CT-UUI system provides fully co-registered anatomical, functional and molecular imaging with high spatial and temporal resolution in small animals. This technology enriches the panoply of parameters that can be mapped simultaneously for the improved assessment of multivariate physiology and complex diseases.

\section{Methods}

Design of the PET-CT-UUI hybrid imaging instrument. The PET-CT-UUI system was assembled by customizing two commercially available instruments-a clinical UUI device (Aixplorer; Supersonic Imagine) and a pre-clinical PET-CT system (nanoScan PET/CT; Mediso) (Supplementary Fig. 1). Two ultrasound transducers were used: a prototype transducer $(15 \mathrm{MHz}$ centre frequency; 128 elements; $100 \mu \mathrm{m}$ pitch) (Vermon) and a commercially available transducer (12 MHz centre frequency; 192 elements; $140 \mu \mathrm{m}$ pitch) (SuperLinear SLH20-6; Supersonic Imagine). Dedicated and customized ultrasound imaging sequences were implemented on the ultrasound scanner to perform UUI and PET-CT imaging simultaneously.

A six-degrees-of-freedom high-precision commercial motorized positioner with a $0.2 \mu \mathrm{m}$ minimum incremental motion (Hexapod H811; Physik Instrumente) was fixed upside down on a hanging bracket made from two aluminium construction rails (XT34; Thorlabs) attached to the PET bed. Ultrasound probes were attached to the motorized positioner by means of a 35-cm-long hollow carbon rectangular cuboid (Polyplan Composites). Elements for the adaptation of the different components were custom-designed and 3D printed (MP05825; MakerBot). This manual set-up allowed for the probe to be installed in an initial position located anywhere within the PET gantry before an acquisition. From this initial position, the motion range of the transducer inside the PET gantry was approximately $36 \mathrm{~mm} \times 40 \mathrm{~mm} \times 40 \mathrm{~mm}$. The effect of the transducer's presence in the PET field-of-view on image quality was measured and the results are included in the Supplementary Materials (Supplementary Fig. 4 and the accompanying text) The main results indicate a modification to the estimate of the true radioactive concentration of $6.4 \pm 2.3 \%(n=3$; Supplementary Fig. $4 \mathrm{~g})$ and small modifications to the noise, recovery coefficient and spill-over (Supplementary Fig. $4 \mathrm{~h}-\mathrm{j}$ ).

\section{Spatial co-registration of the imaging volumes of the PET, CT and UUI} modalities. No fiducial markers were used for the spatial co-registration of the three imaging modalities (PET, CT and UUI). Instead, the affine relationships linking the coordinates systems of the three imaging systems were determined by the following calibration procedure.

Co-registration between PET and CT. The nanoScan PET/CT device intrinsically co-registers PET and CT volumes in a single coordinate system. To measure the accuracy of the PET-CT co-registration, the image quality mouse phantom (part number PH-60-00-42; Mediso), designed according to ref. ${ }^{48}$, was filled with $10.6 \mathrm{MBq}$ of $\left({ }^{18} \mathrm{~F}\right) \mathrm{FDG}$ (Gluscan; Advanced Accelerator Applications) mixed with $200 \mu \mathrm{l}$ of Iopamidol (Iopamiron 300, $300 \mathrm{mg}$ iodine $\mathrm{ml}^{-1}$; Bracco) and placed on the PET-CT dedicated bed in the centre of the field of view. A CT scan was acquired in semi-circular mode at $50 \mathrm{kV}$ using 720 projections, full scan, $300 \mathrm{~ms}$ per projection and a binning of 1:1. The CT was reconstructed using filtered back projection. List-mode PET data were collected for $10 \mathrm{~min}$ and binned using a $5 \mathrm{~ns}$ time window, a 400-600 keV energy window and a 1:5 coincidence mode. PET data were reconstructed using the Tera-Tomo reconstruction engine (3D-ordered subset expectation maximization (OSEM))-based manufactured customized algorithm; Mediso) with expectation maximization iterations, scatter and attenuation correction.

We measured the accuracy of PET-CT co-registration in the region corresponding to the smaller rod of the IQ phantom by taking profiles along two perpendicular directions of the transverse cross-section of the rod and fitting Gaussian curves to each profile. The spatial separation between the centroid of the Gaussian curves fitted to the PET and CT profiles was used as a metric to evaluate the accuracy of the PET-CT co-registration. This procedure was repeated for five evenly spaced positions taken along the axial length of the rod. As a result, the accuracy of the PET-CT co-registration was estimated at $0.049+0.007 \mathrm{~mm}$.

Co-registration between the coordinates system of the motorized positioner and the UUI images. Precise co-registration between UUI and PET requires accurate tracking of the ultrasound probe. The position of the ultrasound probe, and thus of the UUI 3D volume, was controlled with a sub-micrometre accuracy by a set of six variables referring to the positions of the six legs of the motorized micropositioner (Supplementary Fig. 2). The driver of the controller converted the position of the six legs into translational (X, Y and $\mathrm{Z}$ ) and rotational $(\mathrm{V}, \mathrm{U}$ and $\mathrm{W}$ ) motions with respect to the centre of rotation representing the origin of the UUI images, O', positioned by definition at the centre of the ultrasound 
probe. The absolute position of $\mathrm{O}^{\prime}$ with respect to the centre of rotation of the Hexapod O was evaluated for co-registration of UUI and PET as follows. Phantom A consisted of a small water bath with six Gutta Percha points with a tip size of $150 \mu \mathrm{m}$ (ATO Zizine) pointing towards the ultrasound probe and immersed in water. These tips were visible in the UUI images and their local maxima were extracted as point sources with sub-resolution accuracy. For each rotational axis, three UUI volumes of the tips were then acquired at three different angles (that is, $\mathrm{U}=-7,0,7, \mathrm{~V}=-1,0,1$ and $\mathrm{W}=-3,0,3$ ) while the two other rotation variables were set to zero, corresponding to a total of nine acquisitions. Hence, for any tip, the three positions acquired from the three volumes were on the same circle segment in positions defined exactly by the tilt angle of the transducer. Knowing the tilt angles, a simple trigonometric calculation determined the centre of rotation at the intersection of the three radii elevated from the common circle segment. Repeating this calculation for the $\mathrm{U}, \mathrm{V}$ and $\mathrm{W}$ rotational axes then yielded the correct value of the centre of rotation, $\mathrm{O}^{\prime}$, adjusted from the initial estimate $\mathrm{O}_{\text {ini. }}^{\prime}$. The accuracy and precision of the estimated centre of rotation were then evaluated by creating UUI volumes of the tips with different combinations of $\mathrm{U}, \mathrm{V}$ and $\mathrm{W}$ values (that is, $\mathrm{U}=5,0,-5, \mathrm{~V}=-0.4,0.4$ and $\mathrm{W}=1.4,0,-1.4)$ and comparing the positions of the local maxima of the tips with predicted maxima positions. Repetition $(n=17)$ of the measurements yielded a mean error in the definition of the centre of rotation of $0.071 \pm 0.029 \mathrm{~mm}$.

Co-registration between PET-CT and UUI. The registration of PET-CT with UUI was performed using a second phantom (phantom B) in a small water bath with three $80 \mu \mathrm{m}$ copper wires visible in the CT and UUI volumes (Supplementary Fig. 3a). Phantom B was positioned inside the PET-CT gantry and was successively imaged with CT and UUI. The UUI scan consisted of 80 steps in the $\mathrm{X}$ direction with a step size of $0.1 \mathrm{~mm}$. The CT reconstructions $(0.1162 \mathrm{~mm} \times 0.1162 \mathrm{~mm} \times 0.1162 \mathrm{~mm}$ voxel size $)$ were interpolated to match the UUI signal voxel size $(0.1 \mathrm{~mm} \times 0.1 \mathrm{~mm} \times 0.1 \mathrm{~mm})$. The local maxima of the wires were then determined for both the CT and UUI images. These points were used to create a new set of images of density functions of the bivariate normal distribution around the local maxima (20 pixels variance). A total of $240(3 \times 80)$ local maxima corresponding to the wire centres were retrieved from the UUI volume. The coregistration process determined the rotation matrix $\mathrm{R}_{(0,0,0)}$ and a translation vector $\mathrm{T}_{(0,0,0)}$ linking the positions of the centres of mass of the wires:

$$
\mathrm{P}_{\mathrm{CT}}=\mathrm{R}_{(0,0,0)} \mathrm{P}_{\mathrm{UUI}(0,0,0)}+\mathrm{T}_{(0,0,0)}
$$

where $\mathrm{P}_{\mathrm{CT}}=\left\{\mathrm{P}_{\mathrm{CT} 1}, \mathrm{P}_{\mathrm{CT} 2}, \mathrm{P}_{\mathrm{CT} 3} \ldots \mathrm{P}_{\mathrm{CT} 240}\right\}$ and $\mathrm{P}_{\mathrm{UUI},(0,0,0)}=\left\{\mathrm{P}_{\mathrm{UUI}}, \mathrm{P}_{\mathrm{UUI}}, \mathrm{P}_{\mathrm{UUI} 3} \ldots \mathrm{P}_{\mathrm{UUI} 240}\right\}$ are the positions of the local maxima in the $\mathrm{CT}$ and UUI volumes, respectively. To determine $\mathrm{R}_{(0,0,0)}$ and $\mathrm{T}_{(0,0,0)}$, a $3 \mathrm{D}$ intensity-based automatic image registration algorithm was applied to align with the local maxima of the UUI and CT volumes (Supplementary Fig. 3). This co-registration process was performed using the 'imregister' function of the Matlab software (Mathworks), which implements a one-plus-one evolutionary optimization process ${ }^{49}$ using a mutual information metric $^{50}$. In cases of scans including rotations of the ultrasound probe, an extra step was performed. Specifically, since the centre of rotation was set at the origin of the UUI volume (section 2.1), the following relationship holds between the position of the images without rotation $\mathrm{P}_{\mathrm{UUI}(0,0,0)}$ and the position $\mathrm{P}_{\mathrm{UUI}(\mathrm{U}, \mathrm{V}, \mathrm{W})}$ of the images after rotation of the ultrasound probe along the axes $\mathrm{U}, \mathrm{V}$ and $\mathrm{W}$ :

$$
\mathrm{P}_{\mathrm{UUI}(\mathrm{U}, \mathrm{V}, \mathrm{W})}=\mathrm{R}_{\mathrm{W}} \mathrm{R}_{\mathrm{V}} \mathrm{R}_{\mathrm{U}} \mathrm{P}_{\mathrm{UUI}(0,0,0)}
$$

Hence, we have

$$
\mathrm{P}_{\mathrm{CT}}=\mathrm{R}_{(0,0,0)} \mathrm{P}_{\mathrm{UUI}(0,0,0)}+\mathrm{T}_{(0,0,0)}=\mathrm{R}_{(0,0,0)}\left(\mathrm{R}_{\mathrm{W}} \mathrm{R}_{\mathrm{V}} \mathrm{R}_{\mathrm{U}}\right)^{\mathrm{T}} \mathrm{P}_{\mathrm{UUI}(\mathrm{U}, \mathrm{V}, \mathrm{W})}+\mathrm{T}_{(0,0,0)}
$$

And the generalized affine transformation is given by

$$
\mathrm{R}_{(\mathrm{U}, \mathrm{V}, \mathrm{W})}=\mathrm{R}_{(0,0,0)}\left(\mathrm{R}_{\mathrm{W}} \mathrm{R}_{\mathrm{V}} \mathrm{R}_{\mathrm{U}}\right)^{\mathrm{T}}
$$

and

$$
\mathrm{T}=\mathrm{T}_{(0,0,0)}
$$

The accuracy of the registration was measured by acquiring CT and UUI images of the wires at ten different positions and comparing the positions of the registered UUI image maxima with the positions of the CT signal maxima. Repetition $(n=20)$ of the measurements yielded a mean accuracy of the co-registration of $0.095 \pm 0.028 \mathrm{~mm}$

Animal models. Animal experiments were approved by the Comité d'Ethique en Expérimentation Animale de l'Université Paris Descartes and registered by the French Ministry for Higher Education and Research under reference numbers 16-098 and 15-045 for mouse and rat experiments, respectively. All animals were housed in a conventional animal facility under a $12 \mathrm{~h}$ light and $12 \mathrm{~h}$ dark cycle and controlled temperature $\left(24^{\circ} \mathrm{C}\right)$ and humidity (50\%). Animals were fed and watered ad libitum.
Sdhb-deficient tumour model. The generation of the $S d h b^{-/-}$cell line has been described previously ${ }^{24}$. Briefly, $S d h b$-floxed mice were generated at the Mouse Clinical Institute (Illkrich, France). A targeting vector containing Sdhb exon 2 flanked by LoxP sites followed by a neomycin (neo) selection cassette flanked by Frt sites was introduced into P1 (129 S2/SvPas) embryonic stem cells by electroporation. Two positive embryonic stem clones were injected into C57BL/6 J blastocysts and the resulting male chimeras gave germline transmission. These mice were mated to Flipase-expressing mice to delete the Neo cassette. $S d h b^{+/ l o x}$ mice were intercrossed to obtain $S d h b^{\text {lox/lox }}$ mice, and mouse chromaffin cells were isolated from the adrenal medulla of these animals. Nine-week-old female NMRI $\mathrm{nu} / \mathrm{nu}$ (Janvier Labs, France; $n=8$ ) mice were housed at a constant temperature $\left(23^{\circ} \mathrm{C}\right)$ and relative humidity $(60 \%)$ under a regular light/dark schedule. Food and water were available ad libitum. The tumour model was established as previously described ${ }^{25}$. Briefly, tumour fragments, originated from subcutaneous allografts of immortalized mouse chromaffin cells carrying a homozygous knockout of the $S d h b$ gene, were grafted in the dorsal fat pad of eight naïve female nude mice and allowed to grow until the tumour diameter evaluated by caliper reached $5 \mathrm{~mm}$, then imaged longitudinally over around 3 weeks, after which the animals were humanely euthanized. One mouse reached a tumour diameter $>5 \mathrm{~mm}$ on day 12 and was imaged at that time and thereafter (see Fig. 2). The same was the case for four mice on day 14 and thereafter, six mice on day 18 and thereafter and seven mice on days 21 and 25. However, on days 28 and 32, the radioactive decay of fluorine reduced the FDG concentration to a level too low for intravenous injection in the mice and only six mice were imaged on these dates (see Fig. 2).

CCL39 tumour model. The CCL39 cell line was derived from Cricetelus griseus species obtained from the American Type Culture Collection. The respirationdeficient mutant $\left(\mathrm{res}^{-}\right)$and the glycolysis-deficient $\left(\mathrm{gly}^{-}\right)$mutant were generated from WT CCL39 cells as described in refs ${ }^{26-28}$. Briefly, CCL39-WT cells presented with a Warburg type of metabolism (aerobic glycolysis) while the gly- cells were deficient for glycolysis by knockout of the glucose-6-phosphate isomerase (E.C. 5.3.1.9) gene and the res ${ }^{-}$cells were respiration-deficient cells obtained by clonal selection. All CCL39 cells were tested for mycoplasma contamination and found to be negative. Animals were randomly assigned to one of the three groups and ten million cells of WT, gly- or res $^{-}$were implanted in the fat pad of eight-week-old female nude mice ( $n=4$ per group; 12 in total) and allowed to grow to a volume of $500 \mathrm{~mm}^{3}$ before imaging was performed.

Rat model. Ten healthy female Wistar rats weighting approximately $100 \mathrm{~g}$ were obtained from Janvier Labs.

Tumour imaging in mice. No blinding was applied to the experiments. Mice were fasted overnight before the imaging session. During all imaging procedures, mice were anaesthetized with $2.0 \pm 1.0 \%$ isoflurane (IsoVet $100 \%$; Centravet) in $100 \% \mathrm{O}_{2}$. Before the acquisitions, mice were weighed, glycaemia was measured in blood drawn from the ventral tail artery (mean of all exams: $170 \mathrm{mg} \mathrm{dl}^{-1}$ ) using an Accu-Chek Aviva Nano A (Accu-Chek) and the tumour size was measured. A customized catheter with a $26 \mathrm{Ga}$ needle (Fischer Scientific) connected to $5 \mathrm{~cm}$ polyethylene tubing (Tygon Microbore Tubing, 0.010 inch $\times 0.030$ inch OD; Fisher Scientific) was inserted in the tail vein. The body temperature and respiration were monitored. The rectal temperature of the animals was monitored and maintained by controlled heating of the examination bed during the entire procedure.

The animals were positioned in the anaesthesia/imaging bed of the PET-CT scan. Degassed impedance matching gel (MédiGel Clear; Drexco Medical) was applied locally on the skin of the animal and a customized 3D-printed water bath was positioned over the region or organ of interest, affixed to the animal bed and maintained in position for all three scans (PET, CT and UUI). Hence, while the water bath was in contact with the degassed gel applied to the animal, the probe itself was never in contact with the window or the animal and probe motion did not incur any additional motion or deformation of the tissue. The bottom of the water bath was made of a 6- $\mu$ m-thick film of polyethylene terephthalate (ES301061; Goodfellow SARL) to minimize the interface effects between the water and the gel. Imaging was performed after the immersion of the ultrasound probe using the motorized micropositioner (Supplementary Fig. 1). This approach ensured free movement of the ultrasound probe while limiting the risk of creating air bubbles in the path of the ultrasound emissions. The animal bed was moved inside the PET gantry and the motorized positioner was programmed to acquire real-time ultrasound B-mode images to determine the region to image with UUI.

The UUI and PET acquisitions were performed simultaneously. The PET acquisition was set to start $30 \mathrm{~s}$ after injection of $10 \mathrm{MBq}$ of $\left({ }^{18} \mathrm{~F}\right) \mathrm{FDG}$ in $0.2 \mathrm{ml}$ saline into the tail vein of the mice. List-mode PET data were collected between 0 and $60.5 \mathrm{~min}$ post-injection and binned using a $5 \mathrm{~ns}$ time window, a $400-600 \mathrm{keV}$ energy window and a 1:5 coincidence mode (that is, each detector's lines of coincidence with the five opposite detectors). UUI volumes were acquired in $12.8 \times 20 \mathrm{~mm}^{2}$ slices separated by $0.1 \mathrm{~mm}$. The number of joint acquisition planes (range: 70 to 200) was based on the size of the tumour. For each plane, 300 frames were obtained at a rate of 500 frames per second. Each frame was obtained by coherently compounding ${ }^{51}$ the images associated with 11 tilted plane waves equally spaced between -10 and 10 degrees $^{2}$. To minimize motion artefacts, image 
acquisitions were triggered during the respiratory pause of the animal. After the 60-min-long PET acquisition, the animal bed was moved out of the PET gantry and released from the bed. Anaesthesia was discontinued and the animal was allowed to recover on a heating pad.

PET data were reconstructed using the 3D-OSEM-based Tera-Tomo reconstruction engine with expectation maximization iterations and scatter and attenuation correction. Then, 18-frame dynamic images were reconstructed with the following time sequence: exclusion of the first $30 \mathrm{~s}, 4$ frames of $30 \mathrm{~s}, 3$ frames of $60 \mathrm{~s}, 5$ frames of $120 \mathrm{~s}, 3$ frames of $5 \mathrm{~min}$ and 3 frames of $10 \mathrm{~min}$. Standard static images were formed using the last $10 \mathrm{~min}$ of the acquisition (that is, a summed image 50-60 min post FDG injection) using the same reconstruction parameters.

For the UUI reconstructions, each stack of 300 frames was processed to generate an ultrafast power Doppler ${ }^{52,53}$ volume representing the perfused vascular anatomy, for which the amplitude is approximately proportional to the blood volume. Specifically, a spatiotemporal filter based on the singular value decomposition was applied to separate the tissue from the blood signal ${ }^{54}$ and the power (that is, the square of the signal amplitude) was then integrated over the 300 frames, which corresponded to $600 \mathrm{~ms}$ (that is, approximately two heart cycles). The process was repeated for each slice and provided a $3 \mathrm{D}$ volume of the vasculature of the tumour with an anisotropic resolution of approximately $100 \mu \mathrm{m} \times 100 \mu \mathrm{m} \times 400 \mu \mathrm{m}$.

The PET volume was segmented according to the method of ref. ${ }^{55}$. Briefly, a threshold corresponding to $30 \%$ of the SUV peak value was applied on the FDG signal of the tumour area to obtain a volume of interest representing the viable tumour tissue. After the UUI and PET volumes had been co-registered, this volume of interest was transformed into a binary mask and applied to the UUI volume yielding the UUI signal of the viable tumour area. Next, segmentation of the vessels larger than the ultrasound resolution was based on the power Doppler signal. The level of noise was estimated in regions containing ultrasound gel (and thus no vessels) and used to define a threshold equal to twice the noise value.

Cardiac imaging in rats. Combined PET and UUI were performed during several cardiac heart cycles and gated according to the ECG of the animal. Rats were anaesthetized with $2.5 \%$ of isoflurane (IsoVet $100 \%$; Centrevet) in $100 \% \mathrm{O}_{2}$. Rats were weighed, the fur on their chests was removed using depilatory cream and a $23 \mathrm{G}$ catheter (Terumo) was installed in the tail vein. Before imaging, glycaemia was measured using an Accu-Chek Aviva Nano A (Accu-Chek) apparatus.

A custom 3D-printed rat holder was built and adapted to the rat anaesthesia/ holding bed of the PET-CT device (Supplementary Fig. 1c). Accounting for the space constraints due to the ultrasound probe used for cardiac imaging, in this geometry, the anaesthetized rat was positioned in a $60^{\circ}$ Fowler's position. To facilitate the acquisition of standard echocardiographic views, an octopus tissue stabilizer system (Medtronic) was repurposed as a probe holder, first in flexible mode to allow for view search, then in rigid mode once the desired view of the heart had been selected. After positioning, the probe was controlled remotely using the motorized micropositioner.

Rats were positioned on the customized bed and ECG monitoring electrodes were installed (Red Dot; $3 \mathrm{M}$ ). The SLH20-6 probe was positioned on the rat chest to obtain a standard, parasternal short-axis view at the mid-level. A first CT was acquired (semi-circular mode; $37 \mathrm{kV} ; 720$ projections full scan; $300 \mathrm{~ms}$ per projection; binning 1:4). A second CT scan was acquired with the same parameters and the ultrasound probe was positioned out of the PET-CT field of view. A $30 \mathrm{~min}$ gated cardiac acquisition was started $30 \mathrm{~s}$ after injection of $40 \mathrm{MBq}\left({ }^{18} \mathrm{~F}\right) \mathrm{FDG}$ ( $300 \mu \mathrm{l}$ in saline). During the first $30 \mathrm{~min}$ of the PET acquisition, ECG-triggered UUI B-mode imaging using 20 coherently compounded tilted plane waves (equally spaced between -10 and $+10^{\circ}$; pulse repetition frequency of $16,000 \mathrm{~Hz}$ ) was performed. Each high-quality B-mode image was thus obtained within $1.35 \mathrm{~ms}$ and able to track the fast motion of cardiac tissues and detect blood flow in the myocardial vessels. The cardiac shear-wave imaging ${ }^{31}$ mode was also used to quantify the stiffness of the anterior wall. A $300 \mu$ s pushing beam was focused in a region corresponding approximately to the mid-myocardium to generate a shear wave, after which 20 coherently compounded images combining 3 tilted plane waves with angles equally spaced between -10 and $10^{\circ}$ were acquired at 500 frames per second. Shear-wave imaging was performed at ten different time points of the heart cycle using ECG gating. A second PET dataset was collected in list mode between 50 and $60 \mathrm{~min}$ post-injection. Body temperature was maintained at $36-37^{\circ} \mathrm{C}$ and the ECG was monitored and recorded during the entire procedure. After the PET acquisition, the animal bed was moved out of the PET gantry and the animal was released from the bed. Anaesthesia was discontinued and the animal was allowed to recover on a heating pad.

Both PET datasets were binned using a $5 \mathrm{~ns}$ time window, a $400-600 \mathrm{keV}$ energy window and a 1:5 coincidence mode, and reconstructed using the 3D-OSEM-based Tera-Tomo reconstruction engine with expectation maximization iterations and scatter and attenuation correction. Eight-frame gated cardiac images were produced and temporally interpolated to the UUI frame rate and co-registered.

For the imaging of tumours, the probe was placed inside a water bath positioned over the tumour, and respiratory movements could not affect the accuracy of PET-CT-UUI co-registration. In contrast, for the imaging of the heart, the probe was placed in the ultrasound gel layer covering the chest; therefore, the risk that respiratory movements could degrade the accuracy of PET-CT-UUI registration remained. To account for this, the position of the UUI image obtained through the calibration procedure was compared with the CT image of the transducer plate of the probe after it had been positioned in acquisition mode. Whenever the CT-UUI co-registration accuracy was affected, corrections of the co-registration matrix were applied based on the CT image.

To enhance UUI B-mode imaging, incoherent compounding based on the summation of B-mode intensity maps was also applied to a sliding window of ten frames. Ultrafast Doppler imaging of intramyocardial vessels was performed using a previously described methodology ${ }^{29}$ adapted to benefit from the use of a spatiotemporal filter ${ }^{54}$. A spatiotemporal filter based on the truncated singular value decomposition of the stack of 30 demodulated in-phase-quadrature ultrasound images (that is, $40.5 \mathrm{~ms}$ ) was applied to separate the tissue from the blood signal ${ }^{12}$ with a threshold equal to 20 . Images of blood vessels were then obtained by computing the square of the signal amplitude within that 30 -frame window. Shear-wave velocity was estimated using a previously described methodology $y^{31}$. Tissue motion was estimated using in-phase and quadrature frameto-frame cross-correlation. The tissue velocity associated with the shear-wave propagation was isolated by subtracting the average motion of the myocardium. The shear-wave velocity was estimated using a time-of-flight algorithm described in a previous study ${ }^{31}$. Inter-frame displacements were averaged in the thickness of the myocardial wall and the time-of-flight was computed by detecting the maximum temporal correlation between pairs of pixels.

Since the PET volume was associated with a composite heart cycle duration, the duration of the heart cycle was determined in UUI and used to temporally interpolate both datasets to the same heart cycle duration.

Imaging parameters used in UUI. An important trade-off in UUI is the choice of the number of compounded tilted plane waves $N$, which is inversely proportiona to the frame rate and linked to contrast and resolution. $N$ is typically kept small (that is, $\sim 10$ ) to maintain high frame rates. When $N$ is small, improvements in resolution with $N$ are small in comparison to gains in contrast, which thus becomes the main limiting factor along with the frame rate when optimizing a sequence.

For Doppler imaging of small vessels in an organ undergoing a restricted range of motion, exceeding frame rates of 500 frames per second has a limited effect on the image quality. The frame rate (FR) depends on the known speed of sound, $c$, the imaging depth, $d$, the size of the probe, $a$, and the number of compounded plane waves, $N$, as follows: $\mathrm{FR}=\frac{c}{2 N \sqrt{ }\left(d^{2}+a^{2}\right)}$. At a depth of view of $20 \mathrm{~mm}$, this technically enables $N=64$. However, in practice, we observed that $N=11$ provided comparable image quality while limiting the data flow to manageable values as demonstrated by ref. ${ }^{52}$. Moreover, with such a limited number of tilted plane waves, the gain in the ultrasonic image using plane-wave compounding has been shown to typically perform up to 30 times better in terms of sensitivity and three times better in terms of signal-to-noise ratio (SNR) than conventional ultrasound imaging $^{53}$. In other words, due to the small imaging depth, a high image quality was comfortably achieved in a static organ. Hence, in tumours, the main limiting factors were the physiological parameters and, to limit the difficulties inherent to large motion ranges, acquisitions were performed during the respiratory pause, which enables 300 frames and 600-ms-long integration windows for Doppler processing. Assuming a respiratory rate of $1 \mathrm{~Hz}$ in anaesthetized mice, an entire image volume consisting of 200 slices required a (strict) minimum of $200 \mathrm{~s}$. In practice, to account for the motion of the micropositioner and data processing, acquisitions typically lasted 15 to $20 \mathrm{~min}$.

In the heart where a large motion range is inevitable, sequences were heuristically optimized depending on the mode used (B-mode, Doppler or shearwave imaging). These optimizations were typically associated with higher frame rates and a smaller number of compounded plane waves, but also depended on the cardiac image quality, possibly altered by the shadowing and clutter caused by the rib cage.

Statistical analysis. Due to the exploratory nature of this study, the sample size used was not chosen to detect a pre-specified effect size. In rare cases, unexpected vibrations of the building degraded the quality of the power Doppler image. In these cases, the data were excluded from the analysis (note that the consequences of building vibrations have now been eliminated by improving the experimental setup). No blinding was applied. Randomization was used for mice xenografts of the CCL39 cell lines. The data used met the assumptions of the Wilcoxon signed rank test, which are independence (different animals), dependent samples (evolution of parameters in individual animals) and a continuous dependent variable (linked to growth). In the longitudinal mouse tumour study, a paired Student's $t$-test was used to compare the means of the tumour and vessel volumes determined separately for each animal at individual time points.

Life Sciences Reporting Summary. Further information on experimental design is available in the Life Sciences Reporting Summary.

Code availability. The co-registration process described in the section 'Co-registration between PET-CT and UUI' was performed using the 'imregister' 
function from Matlab software (Mathworks). The reconstruction algorithm and data processing methods are described in detail in the Methods. We have opted not to make the data acquisition, image reconstruction and processing code available because the code is proprietary and used for other projects.

Data availability. The data that support the findings of this study are available within the paper and its Supplementary Information. All datasets generated for this study are available from the corresponding author upon reasonable request.

Received: 1 April 2017; Accepted: 23 December 2017; Published online: 6 February 2018

\section{References}

1. Weissleder, R. \& Pittet, M. J. Imaging in the era of molecular oncology. Nature 452, 580-589 (2008).

2. Hanahan, D. \& Weinberg, R. A. Hallmarks of cancer: the next generation. Cell 144, 646-674 (2011).

3. Beyer, T. et al. A combined PET/CT scanner for clinical oncology. J. Nucl. Med. 41, 1369-1379 (2000)

4. Hasegawa, B. H. et al. Dual-modality imaging of cancer with SPECT/CT. Technol. Cancer Res. Treat. 1, 449-458 (2002).

5. Schmand, M. et al. BrainPET: first human tomograph for simultaneous (functional) PET and MR imaging. J. Nucl. Med. 48, 45P (2007).

6. Judenhofer, M. S. et al. Simultaneous PET-MRI: a new approach for functional and morphological imaging. Nat. Med. 14, 459-465 (2008).

7. Mace, E. et al. Functional ultrasound imaging of the brain. Nat. Methods $\mathbf{8}$, 662-664 (2011).

8. Errico, C. et al. Ultrafast ultrasound localization microscopy for deep super-resolution vascular imaging. Nature 527, 499-502 (2015).

9. Sieu, L.-A. et al. EEG and functional ultrasound imaging in mobile rats. Nat. Methods 12, 831-834 (2015).

10. Osmanski, B.-F., Pezet, S., Ricobaraza, A., Lenkei, Z \& Tanter, M. Functional ultrasound imaging of intrinsic connectivity in the living rat brain with high spatiotemporal resolution. Nat. Commun. 5, 5023 (2014).

11. Herholz, K. \& Ebmeier, K. Clinical amyloid imaging in Alzheimer's disease. Lancet Neurol. 10, 667-670 (2011).

12. Han, S. et al. Subregional pattern of striatal dopamine transporter loss on ${ }^{18} \mathrm{~F}$ FP-CIT positron emission tomography in patients with pure akinesia with gait freezing. JAMA Neurol. 73, 1477-1484 (2016).

13. Irkle, A. et al. Identifying active vascular microcalcification by (18)F-sodium fluoride positron emission tomography. Nat. Commun. 6, 7495 (2015).

14. Molecular Imaging and Contrast Agent Database (MICAD) (National Center for Biotechnology Information, Bethesda, MD, 2004); https://www.ncbi.nlm. nih.gov/books/NBK5330/

15. Tanter, M. \& Fink, M. Ultrafast imaging in biomedical ultrasound. IEEE Trans. Ultrason. Ferroelectr. Freq. Control. 61, 102-119 (2014).

16. Tanter, M. et al. Quantitative assessment of breast lesion viscoelasticity: initia clinical results using supersonic shear imaging. Ultrasound Med. Biol. 34, 1373-1386 (2008).

17. Provost, J., Lee, W.-N., Fujikura, K. \& Konofagou, E. E. Imaging the electromechanical activity of the heart in vivo. Proc. Natl. Acad. Sci. USA 108, 8565-8570 (2011).

18. Imbault, M. et al. Robust sound speed estimation for ultrasound-based hepatic steatosis assessment. Phys. Med. Biol. 62, 3582-3598 (2017).

19. Fernández-Sánchez, M. E. et al. Mechanical induction of the tumorigenic $\beta$ -catenin pathway by tumour growth pressure. Nature 523, 92-95 (2015)

20. Demené, C. et al. 4D microvascular imaging based on ultrafast Doppler tomography. NeuroImage 127, 472-483 (2016).

21. Provost, J. et al. 3D ultrafast ultrasound imaging in vivo. Phys. Med. Biol. 59, L1-L13 (2014)

22. Timmers, H. J. L. M. et al. Superiority of fluorodeoxyglucose positron emission tomography to other functional imaging techniques in the evaluation of metastatic SDHB-associated pheochromocytoma and paraganglioma. J. Clin. Oncol. 25, 2262-2269 (2007).

23. Favier, J. et al. The Warburg effect is genetically determined in inherited pheochromocytomas. PLoS ONE 4, e7094 (2009).

24. Letouzé, E. et al. SDH mutations establish a hypermethylator phenotype in paraganglioma. Cancer Cell. 23, 739-752 (2013).

25. Lussey-Lepoutre, C. et al. In vivo detection of succinate by magnetic resonance spectroscopy as a hallmark of SDHx mutations in paraganglioma. Clin. Cancer Res. 22, 1120-1129 (2016).

26. Pouysségur, J., Franchi, A. \& Pagès, G. pHi, aerobic glycolysis and vascular endothelial growth factor in tumour growth. Novartis Found. Symp. 240, 186-196 (2001).

27. Pouysségur, J., Franchi, A., Salomon, J. C. \& Silvestre, P. Isolation of a Chinese hamster fibroblast mutant defective in hexose transport and aerobic glycolysis: its use to dissect the malignant phenotype. Proc. Natl. Acad. Sci. USA 77, 2698-2701 (1980).
28. Franchi, A., Silvestre, P. \& Pouysségur, J. A genetic approach to the role of energy metabolism in the growth of tumor cells: tumorigenicity of fibroblast mutants deficient either in glycolysis or in respiration. Int. J. Cancer 27, 819-827 (1981)

29. Osmanski, B.-F. et al. Ultrafast Doppler imaging of blood flow dynamics in the myocardium. IEEE Trans. Med. Imaging 31, 1661-1668 (2012).

30. Pernot, M. et al. Shear wave imaging of passive diastolic myocardial stiffness: stunned versus infarcted myocardium. JACC Cardiovasc. Imaging 9, 1023-1030 (2016)

31. Pernot, M. et al. Real-time assessment of myocardial contractility using shear wave imaging. J. Am. Coll. Cardiol. 58, 65-72 (2011).

32. Porter, T. R. \& Xie, F. Myocardial perfusion imaging with contrast ultrasound.JACC Cardiovasc. Imaging 3, 176-187 (2010).

33. Papadacci, C., Tanter, M., Pernot, M. \& Fink, M. Ultrasound backscatter tensor imaging (BTI): analysis of the spatial coherence of ultrasonic speckle in anisotropic soft tissues. IEEE Trans. Ultrason. Ferroelectr. Freq. Control. 61 986-996 (2014).

34. Maresca, D. et al. Noninvasive imaging of the coronary vasculature using ultrafast ultrasound. JACC Cardiovasc. Imaging https://doi.org/10.1016/j. jcmg.2017.05.021 (2017).

35. Piert, M. et al. ${ }^{18} \mathrm{~F}$-choline PET/MRI: the additional value of PET for MRI-guided transrectal prostate biopsies. J. Nucl. Med. 57, 1065-1070 (2016).

36. Porporato, P. E., Dhup, S., Dadhich, R. K., Copetti, T. \& Sonveaux, P. Anticancer targets in the glycolytic metabolism of tumors: a comprehensive review. Front. Pharmacol. 2, 49 (2011).

37. Sonveaux, P. et al. Targeting lactate-fueled respiration selectively kills hypoxic tumor cells in mice. J. Clin. Invest. 118, 3930-3942 (2008).

38. Konofagou, E. E., D’hooge, J. \& Ophir, J. Myocardial elastography-a feasibility study in vivo. Ultrasound Med. Biol. 28, 475-482 (2002).

39. Martín, A. et al. Imaging of perfusion, angiogenesis, and tissue elasticity after stroke. J. Cereb. Blood Flow. Metab. 32, 1496-1507 (2012).

40. Ostergaard, L. et al. The relationship between tumor blood flow, angiogenesis, tumor hypoxia, and aerobic glycolysis. Cancer Res. 73, 5618-5624 (2013).

41. Schwaab, J. et al. First steps toward ultrasound-based motion compensation for imaging and therapy: calibration with an optical system and 4D PET imaging. Front. Oncol. 5, 258 (2015).

42. Walker, W. F. \& Trahey, G. E.A fundamental limit on the performance of correlation based phase correction and flow estimation techniques. IEEE Trans. Ultrason. Ferroelectr. Freq. Control. 41, 644-654 (1994).

43. Provost, J., Thiébaut, S., Luo, J. \& Konofagou, E. E. Single-heartbeat electromechanical wave imaging with optimal strain estimation using temporally-unequispaced acquisition sequences. Phys. Med. Biol. 57, 1095-1112 (2012).

44. Hingot, V., Errico, C., Tanter, M. \& Couture, O. Subwavelength motioncorrection for ultrafast ultrasound localization microscopy. Ultrasonics 77, 17-21 (2017).

45. Luo, J. \& Konofagou, E. E.High-frame rate, full-view myocardial elastography with automated contour tracking in murine left ventricles in vivo. IEEE Trans. Ultrason. Ferroelectr. Freq. Control. 55, 240-248 (2008).

46. Lee, W.-N., Provost, J., Fujikura, K., Wang, J. \& Konofagou, E. E. In vivo study of myocardial elastography under graded ischemia conditions. Phys. Med. Biol. 56, 1155-1172 (2011).

47. Denarie, B. et al. Coherent plane wave compounding for very high frame rate ultrasonography of rapidly moving targets. IEEE Trans. Med. Imaging 32, 1265-1276 (2013).

48. Szanda, I. et al. National Electrical Manufacturers Association NU-4 performance evaluation of the PET component of the NanoPET/CT preclinical PET/CT scanner. J. Nucl. Med. 52, 1741-1747 (2011).

49. Styner, M. \& Gerig, G. Evaluation of 2D/3D Bias Correction with 1+ 1ES-Optimization (ETH, Zurich, 1997).

50. Wells, W. M., Viola, P., Atsumi, H., Nakajima, S. \& Kikinis, R. Multi-modal volume registration by maximization of mutual information. Med. Image Anal. 1, 35-51 (1996)

51. Montaldo, G., Tanter, M., Bercoff, J., Benech, N. \& Fink, M. Coherent plane-wave compounding for very high frame rate ultrasonography and transient elastography. IEEE Trans. Ultrason. Ferroelectr. Freq. Control. 56, 489-506 (2009)

52. Bercoff, J. et al. Ultrafast compound Doppler imaging: providing full blood flow characterization. IEEE Trans. Ultrason. Ferroelectr. Freq. Control. 58, 134-147 (2011)

53. Mace, E. et al. Functional ultrasound imaging of the brain: theory and basic principles. IEEE Trans. Ultrason. Ferroelectr. Freq. Control. 60, 492-506 (2013)

54. Demene, C. et al. Spatiotemporal clutter filtering of ultrafast ultrasound data highly increases Doppler and fUltrasound sensitivity. IEEE Trans. Med. Imaging 34, 2271-2285 (2015).

55. Wu, I., Wang, H., Huso, D. \& Wahl, R. L. Optimal definition of biologica tumor volume using positron emission tomography in an animal model. EJNMMI Res. 5, 58 (2015) 


\section{Acknowledgements}

This study was supported by France Life Imaging grant ANR-11-INBS-0006. The authors are grateful to $\mathrm{O}$. Clément and $\mathrm{A}$. Tedgui for constant support, and to $\mathrm{K}$.

Tzavella, G. Leenders, L. Bao and X. Zhang for help with the image co-registration software. This work was supported in part by LABEX WIFI (Laboratory of Excellence ANR-10-LABX-24) within the French programme 'Investments for the Future' under reference ANR-10-IDEX-0001-02 PSL and by a grant from the Plan Cancer Physicancer programme BIMUPET (C16025KS). In vivo imaging was performed at the Life Imaging Facility of Paris Descartes University (Plateforme Imageries du Vivant), supported by France Life Imaging (grant ANR-11-INBS-0006) and Infrastructures Biologie-Santé. The project also received the support of the Institut National de la Santé et de la Recherche Médicale (Inserm) Technology Research Accelerator in Biomedical Ultrasound.

\section{Author contributions}

J.Pr., A.G., M.T. and B.T. designed the experiments. J.Pr., A.G., J.S., D.B., B.B., T.V. and M.P.-L. performed the experiments. J.Pr., A.G., J.S., D.B., B.B., T.V., M.P.-L., M.C., M.P. and B.T. analysed the data. C.L.-L., J.F. and J.Po. provided original material to perform the experiments. J.Pr., A.G., M.T. and B.T. wrote the paper.

\section{Competing interests}

M.T. is a co-founder and shareholder of Supersonic Imagine. All other authors declare no competing financial interests.

\section{Additional information}

Supplementary information accompanies this paper at https://doi.org/10.1038/s41551018-0188-Z

Reprints and permissions information is available at www.nature.com/reprints. Correspondence and requests for materials should be addressed to M.T. or B.T.

Publisher's note: Springer Nature remains neutral with regard to jurisdictional claims in published maps and institutional affiliations. 


\section{natureresearch}

Corresponding author(s): Bertrand Tavitian

Initial submission

Revised version

Xinal submission

\section{Life Sciences Reporting Summary}

Nature Research wishes to improve the reproducibility of the work that we publish. This form is intended for publication with all accepted life science papers and provides structure for consistency and transparency in reporting. Every life science submission will use this form; some list items might not apply to an individual manuscript, but all fields must be completed for clarity.

For further information on the points included in this form, see Reporting Life Sciences Research. For further information on Nature Research policies, including our data availability policy, see Authors \& Referees and the Editorial Policy Checklist.

\section{- Experimental design}

\section{Sample size}

Describe how sample size was determined.

Because of the exploratory nature of this study, the sample size used was not chosen to detect a pre-specified effect size.

2. Data exclusions

Describe any data exclusions.

In rare cases, unexpected vibrations of the building degraded the quality of the power Doppler image. When so, the data was excluded from the analysis. The consequences of building vibrations were later eliminated by improving the experimental setup.

\section{Replication}

Describe whether the experimental findings were reliably reproduced.

4. Randomization

Describe how samples/organisms/participants were allocated into experimental groups.

5. Blinding

Describe whether the investigators were blinded to group allocation during data collection and/or analysis.

Experiments were replicated in multiple animals.

Note: all studies involving animals and/or human research participants must disclose whether blinding and randomization were used.

\section{Statistical parameters}

For all figures and tables that use statistical methods, confirm that the following items are present in relevant figure legends (or in the Methods section if additional space is needed).

n/a $\mid$ Confirmed

$\bigotimes$ The exact sample size $(n)$ for each experimental group/condition, given as a discrete number and unit of measurement (animals, litters, cultures, etc.)

$\triangle$ A description of how samples were collected, noting whether measurements were taken from distinct samples or whether the same sample was measured repeatedly

$\triangle$ A statement indicating how many times each experiment was replicated

The statistical test(s) used and whether they are one- or two-sided (note: only common tests should be described solely by name; more complex techniques should be described in the Methods section)

A description of any assumptions or corrections, such as an adjustment for multiple comparisons

$\bigotimes$ The test results (e.g. $P$ values) given as exact values whenever possible and with confidence intervals noted

A clear description of statistics including central tendency (e.g. median, mean) and variation (e.g. standard deviation, interquartile range)

Clearly defined error bars

See the web collection on statistics for biologists for further resources and guidance. 
Policy information about availability of computer code

\section{Software}

Describe the software used to analyze the data in this study.

We used multiple custom and commercially available software. The co-registration process (described in the section 'Co-registration between PET/CT and UUI') was performed using the 'imregister' function from Matlab ${ }^{\circledR}$ software (Mathworks, USA). The reconstruction algorithm and data processing methods are described in detail in Methods.

For manuscripts utilizing custom algorithms or software that are central to the paper but not yet described in the published literature, software must be made available to editors and reviewers upon request. We strongly encourage code deposition in a community repository (e.g. GitHub). Nature Methods guidance for providing algorithms and software for publication provides further information on this topic.

\section{- Materials and reagents}

Policy information about availability of materials

\section{Materials availability}

Indicate whether there are restrictions on availability of unique materials or if these materials are only available for distribution by a for-profit company.

\section{Antibodies}

Describe the antibodies used and how they were validated for use in the system under study (i.e. assay and species).

10. Eukaryotic cell lines

a. State the source of each eukaryotic cell line used.

b. Describe the method of cell line authentication used.

c. Report whether the cell lines were tested for mycoplasma contamination.

d. If any of the cell lines used are listed in the database of commonly misidentified cell lines maintained by ICLAC, provide a scientific rationale for their use.
No restrictions on the availability of unique materials.

No antibodies were used.

The CCL39 cell line is derived from Cricetelus griseus sp. obtained from American Type Culture Collection (ATCC, Manassas, Virginia).

Authenticated by the commercial source.

All CCL39 cells were tested for mycoplasma contamination and found to be negative.

No commonly misindentified cell lines were used.

\section{- Animals and human research participants}

Policy information about studies involving animals; when reporting animal research, follow the ARRIVE guidelines

\section{Description of research animals}

Provide details on animals and/or animal-derived materials used in the study.
Animal experiments were approved by the Comité d'Ethique en Expérimentation animale de l'université Paris Descartes, and registered by the French Ministry for Higher Education and Research, under reference numbers 16-098 and 15-045 for the mouse and rat experiments, respectively.

Policy information about studies involving human research participants

\section{Description of human research participants}

Describe the covariate-relevant population characteristics of the human research participants.
The study did not involve human research participants. 